EXP2 has dual functionality during the intraerythrocytic stage of infection

\title{
Channeling the vacuole
}

During the blood stages of their life cycle, Plasmodium spp. replicate within a membrane-enclosed parasitophorous vacuole. To evade immune responses and to remodel the red blood cell for parasite survival, protein effectors are transported across the vacuolar membrane by the Plasmodium translocon of exported proteins (PTEX) complex, which is embedded in the vacuolar membrane on the luminal side of the vacuole. Now Ho et al. determine the structure of PTEX, providing insights into the architecture of the complex and how proteins are translocated through it, and Garten et al. discover that EXP2 (a core protein of the complex) serves dual roles as a proteinconducting channel within PTEX and as a channel that facilitates nutrient passage, independent of the complex.

In the first study, the authors purified the endogenous PTEX complex directly from red blood cells infected with the human parasite Plasmodium falciparum and used cryo-electron microscopy (cryo-EM) to determine the structure of PTEX in two functional states ('engaged' and 'resettling') during the export of unfolded protein cargo. PTEX is composed of five proteins (HSP101, PTEX150, EXP2, PTEX88 and TRX2), and HSP101, PTEX150 and EXP2 form the core of the complex. Cryo-EM revealed that the core consists of seven molecules of both PTEX150 and EXP2, and six molecules of the protein-unfolding AAA+ATPase HSP101. The HSP101 molecules at the top of the complex are tethered to a static, funnel shaped pseudoseven-fold-symmetric channel formed by interdigitating EXP2 and PTEX150 molecules. The amino-termini of EXP2 contain a transmembrane helix that inserts into the vacuolar membrane. The transiently associating PTEX88 and TRX2 proteins were not present in the structures.

The HSP101 hexamer was found to have the greatest structural differences between the 'engaged' and 'resettling' states, which allowed the authors to propose a model for how cycling between these two states mediates protein translocation through the channel. In this model, pore loops in HSP101 form two 'hands' that work together to thread the cargo protein through the pore into the host cell cytoplasm. The structures also provide information on crucial interactions between the PTEX complex components that are indispensable for export of effector proteins during the blood stage of the Plasmodium spp. life cycle.

In the second study, Garten et al. provide evidence that $P$. falciparum EXP2 alone acts as a channel for nutrients such as sugars and amino acids during the intraerythrocytic stage and that it is essential for protein export in the PTEX complex. Using a new genetic system to control the translation of EXP2, depletion of EXP2 had severe effects on effector protein export, blood stage survival and vacuole morphology, consistent with an essential role of EXP2 in effector protein export.

As an unknown nutrient channel in the vacuolar membrane had been previously characterized electrophysiologically, the authors tested whether EXP2 could act as a channel for nutrients. In cell-attached patch-clamp experiments, increased expression of EXP2 in cells tightly correlated with the conductance of nutrient ions. The use of an EXP2 mutant that had a truncated carboxyl-terminus (which is

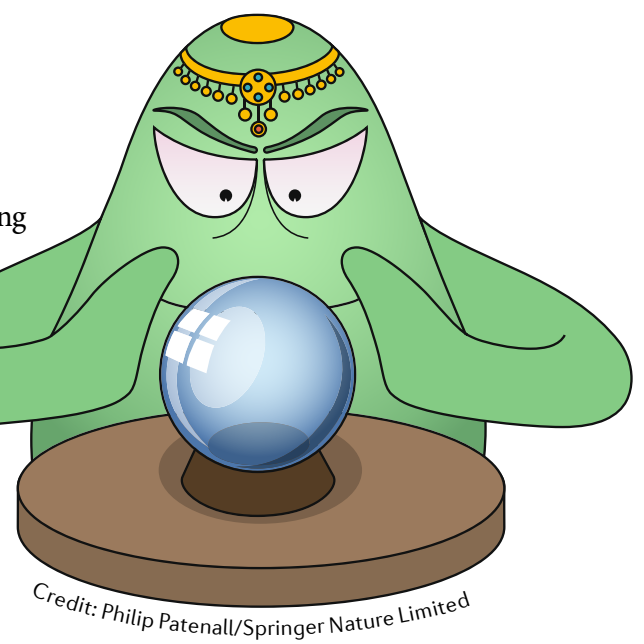

not required for protein export) altered the conductance properties of EXP2, confirming that EXP2 is the nutrient channel. Other experiments suggested that this function is independent of other components of the PTEX complex. Thus, the authors propose that EXP2 has dual functionality during the intraerythrocytic stage of infection.

The notion that EXP2 has a distinct role from its function in the PTEX complex is consistent with the authors' observations that EXP2 expression differs from that of other PTEX complex genes and with their observation that the majority of EXP2 did not co-purify with HSP101 in immunoprecipitation experiments, particularly during the later stages of the life cycle, when EXP2 levels are at their highest.

Together these two studies provide new insights into the architecture, molecular mechanisms and function of the PTEX complex and EXP2, which may lead to the development of new antimalarials.

Ashley York

ORIGINAL ARTICLES Ho, C. et al. Malaria parasite translocon structure and mechanism of effector export. Nature https://doi.org/10.1038/ s41586-018-0469-4 (2018) | Garten, M. et al. EXP2 is a nutrient-permeable channel in the vacuolar membrane of Plasmodium and is essential for protein export via PTEX. Nat. Microbiol. https:// doi.org/10.1038/s41564-018-0222-7 (2018) FURTHER READING de Koning-Ward, T. F. et al. Plasmodium species: master renovators of their host cells. Nat. Rev. Microbiol. 14, 494-507 (2016) 